\title{
Lifespan differences in nonlinear dynamics during rest and auditory oddball performance
}

Viktor Müller ${ }^{1,2}$ and Ulman Lindenberger ${ }^{1,2}$

\author{
1. Center for Lifespan Psychology, Max Planck Institute for Human Development, Germany
}

2. School of Psychology, Saarland University, Germany

\begin{abstract}
Electroencephalographic recordings (EEG) were used to assess age-associated differences in nonlinear brain dynamics during both rest and auditory oddball performance in children aged 9.0-12.8 years, younger adults, and older adults. We computed nonlinear coupling dynamics and dimensional complexity, and also determined spectral alpha power as an indicator of cortical reactivity. During rest, both nonlinear coupling and spectral alpha power decreased with age, whereas dimensional complexity increased. In contrast, when attending to the deviant stimulus, nonlinear coupling increased with age, and complexity decreased. Correlational analyses showed that nonlinear measures assessed during auditory oddball performance were reliably related to an independently assessed measure of perceptual speed. We conclude that cortical dynamics during rest and stimulus processing undergo substantial reorganization from childhood to old age, and propose that lifespan age differences in nonlinear dynamics during stimulus processing reflect lifespan changes in the functional organization of neuronal cell assemblies.
\end{abstract}

\section{Introduction}

As noted by Hebb (1949, p. xiv), 'the problem of understanding behavior is the problem of understanding the total action of the nervous system, and vice versa'. One issue of critical importance in this context is to understand how brain dynamics change across the lifespan. Behavioral and physiological evidence suggests that interactions between maturation and learning from childhood to adulthood lead to increasing cortical differentiation and integration (Nelson \& Luciana, 2001) and that senescent changes from adulthood to old age result in dedifferentiation and reduced cortical specialization of neural cell assemblies (Baltes \& Lindenberger, 1997; Li, Lindenberger \& Sikstrom, 2001; Park, Polk, Park, Minear, Savage \& Smith, 2004; Park, Carp, Hebrank, Park \& Polk, 2010; Park \& Reuter-Lorenz, 2009; Reuter-Lorenz \& Park, 2010). Despite these general claims, surprisingly little is known about lifespan changes in cortical complexity and coupling dynamics. In two earlier articles, we reported lifespan differences in electrophysiological correlates of auditory attention, either by focusing on event-related brain potentials (ERPs; Müller, Brehmer, von Oertzen, Li \& Lindenberger, 2008), or by spectral analyses of evoked power, whole power, and phase synchronization (Müller, Gruber, Klimesch \& Lindenberger, 2009). Both sets of analyses suggested that cortical activity during auditory attention undergoes profound reorganization from childhood to early adult- hood, and from early adulthood to old age. The present article builds on this earlier work by examining lifespan differences in nonlinear brain dynamics during rest and auditory oddball performance.

The human brain is a complex system that shows temporally coherent activity in the absence of an explicit task (Deco, Jirsa, Robinson, Breakspear \& Friston, 2008; Deco, Jirsa, McIntosh, Sporns \& Kotter, 2009; Ghosh, Rho, McIntosh, Kotter \& Jirsa, 2008). This so-called 'resting state' activity and the underlying coupling dynamics relating to it can be captured at different scale levels (from a single cortical area to multiple cortical areas and whole brain dynamics) and frequencies using both neuroimaging techniques (fMRI and PET) and EEG and MEG recordings (Biswal, Yetkin, Haughton \& Hyde, 1995; Damoiseaux, Rombouts, Barkhof, Scheltens, Stam, Smith \& Beckmann, 2006; Deco et al., 2009; Greicius, Krasnow, Reiss \& Menon, 2003; Müller, Birbaumer, Preissl, Braun, Mayer-Kress \& Lang, 2003a; Müller, Preißl, Lutzenberger \& Birbaumer, 2003b; Venables, Bernat \& Sponheim, 2009). Whereas neuroimaging studies have shown synchronous hemodynamic activity at low frequency (ultraslow fluctuations: mostly $<0.1 \mathrm{~Hz}$ ), EEG and MEG studies have shown synchronized electro- or magnetophysiological activity at much higher frequencies $(1-100 \mathrm{~Hz})$. Computational studies (e.g. Deco et al., 2008; Ghosh et al., 2008) suggest that largescale resting state networks are associated with coherent fluctuations that span a wide range of timescales, 
including the timescales captured by imaging and EEG/MEG studies. Computational work also suggests that intrinsic noise and time delays via propagation along connecting fibers contribute to the dynamics of resting state networks (Deco et al., 2008; Ghosh et al., 2008). Therefore, methods and models derived from nonlinear dynamics, such as coupled nonlinear oscillators (Ghosh et al., 2008; Deco et al., 2008), are suitable tools for describing these networks.

Over the past three decades, a number of measures for describing dynamic systems have been introduced (Grassberger \& Procaccia, 1983; Eckmann \& Ruelle, 1985; Wolf, Swift, Swinney \& Vastano, 1985; Ellner, 1988; Briggs, 1990). A common step in attaining such measures consists of reconstructing the phase space by embedding an observable according to Takens' embedding theorem (1981). This theorem proves that the complexity of a dynamic system can be determined by examining the time series of a single observable. The temporal sequence of this observable (like the EEG) thus contains information on all unobserved degrees of freedom. This information can then, in principle, be recovered by reconstructing the phase space from the time series observed in an appropriate manner (Elbert, Ray, Kowalik, Skinner, Graf, and Birbaumer, 1994; Vandenhouten, 1998). One possible way of reconstructing the phase space of a time series is to make use of time-delay embedding (Pritchard \& Duke, 1992; Stam, 2005). Embedding of a time series $x_{t}\left(x_{1}, x_{2}, x_{3}, \ldots, \mathrm{x}_{N}\right)$ is carried out by creating a set of vectors $X_{i}$ such that $X(i)=\left[x_{i}, x_{i+\tau}, x_{i+2 \tau}, x_{i+3 \tau}, \ldots, x_{i+(m-1) \tau}\right]$, where $\tau$ is the time delay in the number of samples and $m$ is the embedding dimension. This embedding procedure allows the evolution of the system to be represented by projecting the vectors $X_{i}$ onto a trajectory in a multidimensional space, often called the phase space or state space. The trajectory or subspace upon which the system converges is called attractor, which can be described mathematically by its dimension (van Drongelen, 2007).

If we assume that each cortical network has its own characteristic temporal dynamics as expressed in the EEG trace, then the dimension of the time series can be interpreted as an estimate of the number of independently activated cell assemblies (Birbaumer, Flor, Lutzenberger \& Elbert, 1995; Elbert et al., 1994; Lutzenberger, Preissl \& Pulvermuller, 1995), which correspond to the cortical complexity of a given mental, motor, or sensory activity. Interacting neurons in the brain dynamically self-organize into coherently oscillating structures that are generated and activated by input from external or internal sources (Mayer-Kress, 1998). Based on a simulation study, Lutzenberger et al. (1995) noted that dimensional complexity increases with the number of independently active generators of spontaneous electro-cortical activity. Whether these independent generators are related to the number of independently active cell assemblies in the Hebbian sense as described by Elbert et al. (1994) remains unclear. According to
Ashby's (1958) Law of Requisite Variety, the variety in the control system must be equal to or larger than the variety of the perturbations to maintain stability. This suggests that a greater degree of complexity in brain activity is needed to keep the system within stable activity boundaries.

Anokhin, Müller, Lindenberger Heath, and Myers (2006) found in a recent study that individual differences in the complexity of resting electrocortical dynamics are largely determined by genetic factors. With regard to developmental changes in the brain's dynamic complexity, there is evidence for increasing complexity from childhood to old age (up to 60 years; Anokhin, Birbaumer, Lutzenberger, Nikolaev \& Vogel, 1996; Anokhin, Lutzenberger, Nikolaev \& Birbaumer, 2000). In terms of changes in complexity with respect to aging, both increases and reductions in complexity have been reported (for review, see Vaillancourt \& Newell, 2002). Pierce, Kelly, Watson, Replogle, King and Pribram (2000) used recrudescence rate and algorithmic complexity measures to show significantly higher levels of EEG complexity in older adults than in younger ones at rest with eyes both closed and open as well as in a sustained attention task. In other work applying factor analysis to EEG data, Pierce, Watson, King, Kelly and Pribram (2003) showed that EEG recordings of older adults yielded significantly more factors than those of younger adults, indicating a greater degree of complexity in the spatial distribution of EEG activity in older than in younger adults. Furthermore, there are also indications that the quantification of EEG complexity and nonlinear coupling may aid the early diagnosis of dementia (Czigler, Csikós, Hidasi, Gaál, Csibri, Kiss, Salacz \& Molnár, 2008; Jelles, van Birgelen, Slaets, Hekster, Jonkman \& Stam, 1999; Jeong, 2004).

Dynamic complexity variations have also been observed during task performance using pointwise measures, reflecting the specific requirements of stimulus processing. Rapp, Bashore, Martinerie, Albano, Zimmerman and Mees (1989) found reduced complexity (D2) in response to the target stimulus compared with the nontarget stimulus during the auditory oddball task. Molnar, Skinner, Csepe, Winkler and Karmos (1995) also reported reductions in dimensional complexity (PD2i, pointwise correlation dimension) in response to the target stimulus in the auditory oddball task relative to baseline, with a stronger reduction in complexity observed for attended stimuli than for ignored stimuli. These findings provide evidence that stimulus processing is associated with a reduction in dynamic complexity. This could be related to a reduction in the system's degrees of freedom due to decision-making or other types of cognitive demand during stimulus processing.

Another important dimension of complex systems is their coupling dynamics. Each subsystem has its own dynamics, and the complex behavior of the whole system reflects, in part, the coupling mechanisms among the subsystems. According to Hebb's theory of cell 
assemblies, the exchange of information between cell assemblies is much smaller than the information flow within each assembly (cf. Buzsáki, 2006; Hebb, 1949; Watts, 2003). This differentiation in the information flow assumes that different coupling dynamics exist between different neural units. These dynamics and their changes over the lifespan can be assessed by both linear and nonlinear measures. Using phase synchronization measures (Phase Locking Index and Phase Coherence), we recently reported that cortical synchronization increased from childhood to old age (Müller et al., 2009). In the present study, we use two nonlinear coupling measures, the Pointwise Transinformation Index (PTI) and the Pointwise Conditional Coupling Divergence (PCCD) as well as a complexity measure (Pointwise Dimension, PD2) to examine how coupling dynamics and complexity change over the lifespan. PTI is derived from Shannon's information concept (Shannon \& Weaver, 1949) and measures the mean quantity of information in a system contained within the other system (Vandenhouten, 1998). As such, it predicts and distinguishes one system's behavior from that of another and can reflect the nonlinear coupling dynamics between the systems. PCCD provides a quantification of dissipative coupling dynamics by observing a system's trajectories over a common phase space, thus indicating how long the two trajectories of the two different systems remain in neighborhood, that is, are dynamically coupled. While some evidence of increasing complexity with age exists, developmental aspects of nonlinear coupling have not been investigated at all. Furthermore, there is no information about the way in which dynamic complexity and nonlinear coupling during stimulus processing differ by age.

Here, we present data from younger children, older children, younger adults, and older adults obtained at rest and during an auditory oddball task under an attended (i.e. oddball-counting) condition. We expected differences in mean complexity and coupling as a function of age group, at least up to early adulthood. Dimensional complexity, which reflects the complexity of the signal and is related to the number of independently activated cell assemblies, was expected to increase with age during rest, accompanied by an age-related increase in the its reduction from rest to task. These expectations are based on the assumption that the complexity of brain dynamics may indicate either of two age-graded changes: (a) a process of increasing cortical differentiation and integration from childhood to adulthood (cf. McIntosh, Kovacevic, Lippe, Garrett, Grady \& Jirsa, 2010); and (b) the disaggregation of functional units due to senescent changes from early to late adulthood (cf. Pierce et al., 2000, 2003). Furthermore, functional complexity reduction during task performance may reflect the efficiency with which the system's degrees of freedom are reduced during stimulus processing. We also expected that nonlinear coupling reflecting information exchange within and between cell assemblies would increase with age, especially during active stimulus processing. In addition, we examined the association between nonlinear measures and behavioral performance on cognitive tests assessing perceptual speed to aid the functional interpretation of dynamic complexity and nonlinear coupling. Given that individual differences on measures of perceptual speed can be interpreted as measures of processing efficiency, we expected perceptual speed scores to be positively related to coupling measures and negatively related to the complexity measure both computed during task condition.

\section{Method}

\section{Participants}

All participants were volunteers recruited via announcements at schools in the Saarland (Gymnasium - the toptier German high school) and at the Saarland University. The older adults were senior students at Saarland University, participated in other continuing education programs, or both. All participants were paid 7.50 Euro per hour to take part in the study. They were all right-handed, had no reported history of head injuries or neurological disorders, and were not on medication. Of the participating individuals, five younger children, one older child, and one older adult were excluded from the data analysis due to their having reported numbers of odd stimuli in attended conditions (see below) that deviated from the correct number by more than three digits in either direction. One younger adult was excluded from the data analysis because of a technical problem in the rest condition. The effective sample thus consisted of 24 younger children $(\mathrm{YC}$, mean age $=9.9, S D=0.6$, age range $=9.0$ 10.8 years, 13 females), 28 older children (OC, mean age $=12.0, S D=0.6$, age range $=11.0-12.8$ years, 14 females), 30 younger adults (YA, mean age $=22.6$, $S D=1.6$, age range $=18.8-25.1$ years, 14 females $)$, and 28 older adults (OA, mean age $=67.8, S D=3.0$, age range $=63.9-74.5$ years, 14 females). Participants of all ages including children were able to sustain their attention for the entire duration of the experiment.

\section{Psychological and audiological assessment}

Psychological and auditory assessments were carried out on the day before the EEG session. The cognitive battery of the Berlin Aging Study (BASE; Baltes \& Mayer, 1999) was used for psychological assessment. Three tests from this battery, namely Digit Symbol Substitution (DSS), Digit Letter Substitution (DLS) and Identical Pictures (IP), all of which comprise marker tests of perceptual speed, were selected for correlational analysis of relationships with electrophysiological data. The materials and procedural details of this cognitive battery have been described elsewhere (Lindenberger, Mayr \& Kliegl, 1993).

The Wechsler (1955) version of the DSS test was used. The participants were presented with a coding key which paired nine numbers (1 through 9 ) with nine symbols 
together with a row of randomly ordered numbers with empty boxes below them. Participants had to enter as many symbols as possible into the empty boxes based on the digit-symbol associations specified in the coding key within $90 \mathrm{~s}$. The number of symbols correctly entered into the boxes represented the outcome measure.

The DLS test closely resembles the DSS but uses letters instead of symbols. The test consists of a total of 21 sheets, each of which contained six digits with a question mark underneath. Moving from left to right, subjects were asked to name the letters corresponding to the digits. The test lasted for 3 minutes, with scores collected after each minute. The score used here was the total number of correct responses after $3 \mathrm{~min}$.

In the IP test, a total of 32 items was presented. For each item, a target figure was presented at the top of the screen together with five response alternatives at the bottom. Participants were asked to touch the correct alternative (i.e. the identical one) at the bottom of the screen as quickly as possible. The subjects were both given instructions and allowed to complete three practice rounds before the actual test phases began. Testing finished automatically after $80 \mathrm{~s}$. The score refers to the number of correct responses.

The standard Hughson-Westlake technique for measuring the pure-tone detection threshold $(0.25-8.0 \mathrm{kHz}$, bilaterally) was used for auditory assessment (Carhart \& Jerger, 1959). The threshold values for left and right sites were averaged and a two-way repeated measures ANOVA with a between-subjects factor Age and a within-subject factor Frequency $(4 \times 8)$ was carried out. Fischer's LSD test was used for post-hoc testing of age-group differences. Statistical analysis revealed a significant main effect for the factors Age, $F(3,107)=47.0, p<.0001$, $\eta^{2}=0.57$, and Frequency, $F(7,749)=18.1, p<.0001$, $\eta^{2}=0.15$, as well as a significant Age by Frequency interaction, $F(21,749)=30.1, \quad p<.0001, \quad \eta^{2}=0.46$. Fischer's LSD post-hoc tests revealed significant differences between OA and other age groups (all $p<.0001$ ). Auditory thresholds were highest in OA and generally lowest in YA. Thresholds were particularly elevated at high frequencies for OA. Age differences were least pronounced in the frequency range of stimuli used for the oddball task (800-1000 Hz).

\section{Procedure}

The EEG measurement began with a 3-minute relaxation phase (1.5 minutes with eyes closed and 1.5 minutes with eyes open). Instructions for the resting states were given on the computer display and were presented as follows: 'A cross will be shown in the middle of the screen for a minute and a half. Please focus on the cross and relax' (for the eyes-open condition) and 'Keep your eyes closed for a minute and a half and relax' (for the eyes-closed condition). The rest phases were then followed by the auditory oddball task. During the recording, the subjects sat in a chair in a relaxed position in an electrically shielded room. During the oddball task, which was carried out with eyes closed, the participants heard two different types of tone pips: a $1000 \mathrm{~Hz}$ tone played frequently to form the standard stimulus and an $800 \mathrm{~Hz}$ tone played only intermittently to form the deviant stimulus. The standard and deviant stimuli were presented binaurally (with a probability of 0.8 and 0.2 for the standard and deviant stimuli, respectively) through headphones (Sony DJ MDR-V300) at $70 \mathrm{~dB}$ SPL for a duration of $70 \mathrm{~ms}$ (including a $10 \mathrm{~ms}$ rise and fall period). The stimuli were generated using the Audacity 1.2.4 software. The inter-stimulus interval ranged between 1200 and $1500 \mathrm{~ms}$. Two different experimental conditions were used: passive listening (unattended) and active counting (attended). For the first condition, the subjects were simply asked to listen to the tone pips without any response, whereas, for the second condition, the subjects were asked to listen to the stimuli and count the number of deviant tones. They were then asked to report back the number of tones counted once the session was complete. Each experimental condition contained 152 standard tones and 38 deviant tones presented in a pseudo-random order fixed for all participants. The conditions were always presented in the same order, with the passive listening condition followed by the active counting condition in order to facilitate the interpretation of between-person differences.

\section{EEG recordings and analyses}

The electroencephalogram (EEG) was recorded from 58 $\mathrm{Ag} / \mathrm{AgCl}$ electrodes using an elastic cap (Electrocap International) with a sampling rate of $500 \mathrm{~Hz}$ in a frequency band ranging between 0.5 and $100 \mathrm{~Hz}$. The left mastoid was used as a reference and the right mastoid was recorded as an active channel. The data were also re-referenced off-line to an average of the left and right mastoids for further analysis. The electrodes were placed according to the international $10-10$ system. For data analyses using nonlinear algorithms, only 21 electrode locations from the 10-20 system were used to avoid volume conduction effects between electrode sites located close together. The vertical and horizontal electrooculogram (EOG) was recorded for control of eye blinks and eye movements. Signals were digitally filtered off-line (Butterworth zero phase filters $0.5-50 \mathrm{~Hz}$, slope $12 \mathrm{~dB} /$ octave; notch filter $50 \mathrm{~Hz}$ ). The EEG recordings were corrected for eye movements using the Gratton and Coles algorithm (Gratton, Coles \& Donchin, 1983), and blink artifacts were rejected based on a gradient criterion (i.e. based on a maximally allowed voltage step of $50 \mu \mathrm{V}$ ), and a difference criterion (i.e. based on a maximally allowed absolute difference between two values in the segment of $200 \mu \mathrm{V}$ ). The EEG was segmented based on division in equal sized non-overlapping segments of $1024 \mathrm{~ms}$ length (512 data points) for the rest interval. During the task, the segmentation was linked to the stimulus-onset, where the segments also contained 512 
data points of the post-stimulus interval. All measures were thus calculated for 30 artifact-free 1024 ms EEG segments chosen randomly after artifact rejection and then averaged across the trials. The data were also averaged across subjects within the age groups (grand averages) for representation purposes.

\section{Nonlinear measures}

The pointwise correlation dimension (PD2) and the two coupling measures - pointwise transinformation (PTI) and pointwise conditional coupling divergence (PCCD) were calculated using the Dataplore software package (Datan Software and Analysis GmbH, Teltow, Germany). All of the pointwise measures used are well suited to examine non-stationary time series and can also be applied to EEG and other biological signals (Vandenhouten, 1998; Elbert et al., 1994).

\section{Pointwise Dimension (PD2)}

The software package uses Skinner's (Skinner, Molnar, Vybiral \& Mitra, 1992; Skinner, Pratt \& Vybiral, 1993) algorithm to determine PD2, which is computed using the following formula: $P D 2(i)=\log C(r, i) / \log (r)$, with the pointwise correlation integral calculated based on:

$$
C(r, i)=\frac{1}{N-1} \sum_{j=0 ; j \neq i}^{N-1} \theta\left(r-\left\|\hat{x}_{i}-\hat{x}_{j}\right\|\right)
$$

where $r$ denotes the radius of a phase-space neighborhood around $x ; \hat{x}_{i}$ und $\hat{x}_{j}$ are the phase space coordinates with delay $\tau ; N$ is the length of the signal, and $\theta$ is the Heavyside function, obtained by:

$$
\theta(x)= \begin{cases}0 & \text { if } x<0 \\ 1 & \text { if } x \geq 0\end{cases}
$$

The PD2 value refers to the dimension of an attractor of the reconstructed time series, thereby yielding a measure of the system's dynamic complexity. The EEG time series were reconstructed with a maximum embedding dimension of 16 and a time delay of $2 \mathrm{~ms}$.

\section{Pointwise Transinformation (PTI)}

PTI is a measure of nonlinear coupling between two time series that is derived from Shannon's information concept (Shannon \& Weaver, 1949). It is calculated on the basis of the probability density of the observables in the multidimensional phase space (Lambertz, Vandenhouten, Grebe \& Langhorst, 2000; Vandenhouten, 1998):

$$
P T I(\tau, r, i)=\log \frac{P_{i}^{\xi \chi}(\tau, r)}{P_{i}^{\xi}(r) P_{i}^{\chi}(r)}
$$

The phase-space densities were assessed using point frequencies $P_{i}(r)$ that were each determined within a sphere of radius $r$ around the points $x_{i}$ of the trajectory reconstructed in the phase space. In simplified terms, the PTI for the $i$-th time point, lag $\tau$, und radius $r$ shows the probability of being able to observe the value of the observable $\chi$ of the second system based on the value $\xi$ of the first system after time $\tau$. Thus, the transinformation measures the mean information quantity of a variable $\xi$ that is contained in the other variable $\chi$ (Vandenhouten, 1998). As phase-space densities are only determined based on empirical point frequencies, PTI can also take on negative values. PTI variations indicate the degree of dynamic coupling between systems, with more positive values corresponding to stronger coupling.

\section{Pointwise Conditional Coupling Divergence (PCCD)}

PCCD is also a measure of nonlinear coupling and provides a quantitative expression of dissipative coupling dynamics (Vandenhouten, 1998; Lambertz et al., 2000). It is based on the assumption that two points $\left(\xi_{i}, \chi_{i}\right)$ and $\left(\xi_{j}, \chi_{j}\right)$ in coupled systems that are close to each other within the common phase space remain close together after a short time interval $t_{\mathrm{e}}=1 \bullet \mathrm{t}_{\mathrm{a}}$. The PCCD is thus defined as an expectation value for the conditional probability that a point in a common phase space is still in a neighborhood with radius $r$ of the reference trajectory after $l$ time steps (Vandenhouten, 1998). PCCD can be calculated according to the formula (Lambertz et al., 2000; Vandenhouten, 1998):

$$
\begin{aligned}
& \operatorname{PCCD}(r, l, i) \\
& =\frac{\sum_{j=0}^{N-1} \theta\left(r-\left\|\left(x_{j}, y_{j}\right)-\left(x_{i}, y_{i}\right)\right\|\right) \theta\left(r-\left\|\left(x_{j+l}, y_{j+l}\right)-\left(x_{i+l}, y_{i+l}\right)\right\|\right)}{\sum_{k=0}^{N-1} \theta\left(r-\left\|\left(x_{k}, y_{k}\right)-\left(x_{i}, y_{i}\right)\right\|\right)}
\end{aligned}
$$

where $x_{i}, y_{i}$ and $x_{j}, y_{j}$ are the coordinates of the two trajectories in phase space, and $\theta$ is the Heaviside function. In contrast to PTI, PCCD is normalized to values between 0 and 1 .

\section{Spectral alpha power}

In addition to nonlinear measures, we also determined the spectral power in the alpha frequency band $(8-12 \mathrm{~Hz})$, primarily as a treatment to validate the manipulation of the eyes-closed vs. eyes-open condition. Spectral alpha power related to the differences between the eyes-open and eyes-closed conditions is also an indicator of reactivity and brain activation (Klimesch, 1999). Alpha power was determined separately for each trial and for each electrode location using fast Fourier transformation (FFT) with Hanning temporal windows and then averaged over trials within experimental conditions (eyes closed vs. eyes open). Power values were normalized using a logarithmic transformation. 


\section{EEG data reduction and statistical analyses}

All of the measures described above were calculated separately for each trial and then averaged across trials. Complexity and nonlinear coupling values obtained during the rest conditions were also averaged over all of the values determined in the sequence. These averages were calculated separately for each electrode or electrode pair and then used for statistical analyses comparing the eyes-closed and eyes-open conditions across ages. We subtracted rest complexity and coupling values from corresponding task values for each time step in order to obtain task-related changes. The resulting difference values were presented as time diagrams for each electrode or electrode pair averaged across participants in the different age groups. For statistical analyses, the difference values for each participant were averaged around the peaks, which were determined in the grand averages as maximum values separately for each age group. Twenty-one electrode locations of the 10-20 electrode system (Fp1, Fpz, Fp2, F7, F3, Fz, ..., Pz, P4, P8, O1, $\mathrm{Oz}, \mathrm{O} 2)$ were used for the statistical analysis of the complexity measure (PD2). Nonlinear coupling measures were first determined for 76 different electrode pairs, which were then collapsed into seven different topological components: AP (anterior to posterior), PA (posterior to anterior), LR (left to right $=$ right to left), WL (within left), WR (within right), $\mathrm{FzO}$ ( $\mathrm{Fz}$ to all other electrode locations), and $\mathrm{PzO}$ (Pz to all other electrode locations). In the time domain, the difference values were averaged separately for each age group around the three peaks determined based on the grand averages. The first two peaks were averaged across the time interval of $40 \mathrm{~ms}$ around the peak and the third peak was averaged across the time interval of $60 \mathrm{~ms}$ around the peak.

\section{Surrogate data test}

The purpose of the surrogate data procedure is to test whether we can refute the null hypothesis that a Gaussian (linear) process has generated the data, thereby providing indirect evidence in favor of the alternative assumption that the data originate from a chaotic system (Theiler, Eubank, Longtin, Galdrikian \& Farmer, 1992). The procedure consisted of: (a) computing the amplitude and phase spectrum of a real signal using a Fourier transformation; (b) data shuffling, whereby the phase values of the original spectrum are used in random order and the sorted values of the surrogate sequence are replaced with the corresponding sorted values of the reference sequence; and (c) reverse Fourier transformation back to the time domain. In this way, the real and the surrogate data $(\mathrm{RD}$ and $\mathrm{SD}$ ) retain the same power spectrum but a different time course. According to the null hypothesis, the RD and the SD are identical in the sense that both can be described by a linear stochastic model, i.e. the two time series will not differ reliably. The null hypothesis is thus rejected if there are significant differences between the correlation dimensions of the $\mathrm{RD}$ and the SD. The surrogate data test was applied for the eyes-closed resting state.

\section{Statistical analyses}

At first, we compared the complexity of real data (RD) with the complexity of the corresponding surrogate data (SD) using a three-way repeated measures ANOVA with a between-subjects factor Age (four age groups: YC, OC, YA, and OA) and two within-subject factors Data (RD vs. SD) and Electrodes (21 electrode locations) to decide whether the analyzed EEG signals were generated from a chaotic system. This analysis was performed for the eyesclosed rest condition. Thereafter, both the nonlinear measures and the spectral alpha power were analyzed for age-related differences during the resting state, comparing the eyes-closed with the eyes-open condition. A three-way repeated measures ANOVA with a betweensubjects factor Age and two within-subject factors Eyes (closed vs. open) and Electrodes was used for both the spectral alpha power and the complexity measure, which were each determined separately for each electrode location. The nonlinear coupling measures were tested using a three-way repeated measures ANOVA with a between-subjects factor Age and two within-subject factors Eyes and Topological Components = TC (seven components: AP, PA, LR, WL, WR, FzO, and PzO). In order to test the task-related changes across the lifespan, the obtained difference values of the nonlinear measures (see above) were subjected to a three-way repeated measures ANOVA Age $\times$ Peaks $\times$ Electrodes for dynamic complexity and to a three-way repeated measures ANOVA Age $\times$ Peaks $\times$ Topological Components for nonlinear coupling measures (PTI and PCCD). The Peaks factor always varied on the three levels, indicating the presence of three different peaks for these measures during the time sequence or trial. Greenhouse-Geisser epsilons were used in all ANOVAs for non-sphericity correction when necessary. Fischer's LSD test was employed for post-hoc testing of age-group differences.

To assess correlations between cognitive performance and electrophysiological data, Pearson product correlations were computed separately for each age group between the performance (raw scores) in the three perceptual speed tasks (DSS, DLS, and IP) and the nonlinear EEG measures during the task condition. To this end, the three performance measures were $z$-transformed and combined by averaging to the one integrative measure of perceptual speed for each age group separately.

\section{Results}

\section{Surrogate data testing}

PD2 values of real and surrogate data were subjected to a three-way repeated measures ANOVA (Age $\times$ Data $\times$ Electrodes), which revealed a significant main effect for 
the Data factor: $F(1,106)=31.8, p<.0001, \eta^{2}=0.23$, with significantly higher PD2 values for surrogate data compared with real data. The Data factor showed a significant level of interaction with the Electrodes factor, $F(20,2120)=11.6, \quad p<.0001, \quad \eta^{2}=0.10$, but not with the Age factor, $F(3,106)=1.8, p=.15, \eta^{2}=0.05$. The interaction Age by Data by Electrodes was significant, $F(60,2120)=2.0, p<.05, \eta^{2}=0.05$, indicating age differences regarding data (real vs. surrogate) and electrodes. The results thus showed that the null hypothesis, that is, that the data are generated by a Gaussian (linear) process, can be rejected and that the EEG time series of the participants can be assumed to be nonlinear in all age groups.

\section{Lifespan differences in EEG dynamics during rest}

Age-related changes for nonlinear coupling (PTI and PCCD) and complexity (PD2) measures during the eyes-closed and eyes-open rest conditions are displayed in Figure 1. It can be seen that: (a) nonlinear coupling (PTI and PCCD) in all age groups was higher when the subjects' eyes were closed than when they were open, whereas the reverse pattern was found for complexity; (b) PTI and PCCD decreased with age whereas PD2 increased with age, both for the eyes-closed and eyesopen conditions. These effects of age group were statistically reliable (see Table 1). The post-hoc Fischer's LSD test for PTI-values showed significantly lower coupling in older adults than in children and younger adults $(\mathrm{OA}<\mathrm{YA}<\mathrm{OC}, \mathrm{YC}$; all $p \mathrm{~s}<.0001)$. With regard to PCCD, nonlinear coupling was significantly lower in adults than in children and also lower in older adults compared with younger adults (all $p \mathrm{~s}<.01$ ). There was also a significant Age-by-Eyes interaction with regard to the PTI measure, indicating a stronger decrease in coupling with an increase in age in the eyes-open than in the eyes-closed condition. In clear contrast to the


Figure 1 Lifespan differences in nonlinear coupling and complexity during rest. (a). Grand average brain maps of nonlinear coupling (PTI and PCCD) and dimensional complexity (PD2) for eyes closed (EC) and open (EO) by age group; (YC) younger children, (OC) older children, (YA) younger adults, and (OA) older adults. All brain maps use the same scaling across ages. Nonlinear coupling decreases and dimensional complexity increases with age. (b). Mean values and standard error bars are displayed for corresponding measures in bar charts for the four age groups and eyes-closed (EC) and eyes-open (EO) conditions. 
Table 1 ANOVA results (F- and p-values) for nonlinear measures (PTI, PCCD and PD2) and alpha spectral power during rest conditions with eyes closed and open

\begin{tabular}{|c|c|c|c|c|}
\hline \multirow[b]{2}{*}{ Measures } & \multicolumn{4}{|c|}{ Age-related effects and interactions } \\
\hline & Age $(\mathrm{df}=3,106)$ & $\begin{array}{l}\text { Age } \times \text { Eyes } \\
(\mathrm{df}=3,106)\end{array}$ & $\begin{array}{c}\text { Age } \times \mathrm{TC}(\mathrm{df}=18,636) \\
\text { Age } \times \text { Electrodes }(\mathrm{df}=60,2120)\end{array}$ & $\begin{array}{c}\text { Age } \times \text { Eyes } \times \text { TC }(\mathrm{df}=18,636) \\
\text { Age } \times \text { Eyes } \times \text { Electrodes }(\mathrm{df}=60,2120)\end{array}$ \\
\hline PTI & $\begin{array}{l}11.4\left(p<.0001, \eta^{2}=0.24\right) \\
\text { YC,OC,YA > OA }\end{array}$ & $\begin{array}{c}3.0(p<.05 \\
\left.\eta^{2}=0.079\right)\end{array}$ & $0.9(n s)$ & $0.5(n s)$ \\
\hline PCCD & $\begin{array}{l}22.9\left(p<.0001, \eta^{2}=0.39\right) \\
\text { YC,OC }>\text { YA }>\text { OA }\end{array}$ & $1.1(n s)$ & $1.2(n s)$ & $0.7(n s)$ \\
\hline PD2 & $\begin{array}{l}29.4\left(p<.0001, \eta^{2}=0.45\right) \\
\text { YC,OC }<\text { YA }<\text { OA }\end{array}$ & $1.8(n s)$ & $2.5\left(p<.001, \eta^{2}=0.066\right)$ & $2.0\left(p<.034, \eta^{2}=0.052\right)$ \\
\hline $\begin{array}{l}\text { Spectral } \\
\text { alpha power }\end{array}$ & $\begin{array}{l}29.9\left(p<.0001, \eta^{2}=0.46\right) \\
\text { OC, }, \mathrm{YC}>\mathrm{YA}>\mathrm{OA}\end{array}$ & $\begin{array}{l}7.8(p<.0001 \\
\left.\eta^{2}=0.18\right)\end{array}$ & $3.6\left(p<.0001, \eta^{2}=0.093\right)$ & $1.3(n s)$ \\
\hline
\end{tabular}

Note: $n s=$ not significant; $\mathrm{TC}=$ Topological Components.

coupling measures, the complexity measure (PD2) showed a significant increase in dimensional complexity with age (see Table 1). Post-hoc tests showed that complexity was significantly higher in adults than in children and also higher in older adults than in younger adults (all $p$ s $<.01$ ). In addition, there were reliable Age by Electrodes and Age by Eyes by Electrodes interactions, indicating different topological distributions of the complexity measure at different ages, and stronger differences in topological distributions with age in the eyes-open than in the eyes-closed condition.

Spectral alpha power changes with age for the eyesclosed and eyes-open conditions are shown in Figure 2. A strong alpha depression was observed for the eyes-open compared with the eyes-closed condition. Also, spectral alpha power decreased with age for both conditions. The frequency diagrams for these two conditions at the $\mathrm{Oz}$ electrode and the corresponding power distributions at the peak frequency are also displayed in Figure 2. It can be seen that: (a) alpha power was generally higher in the eyes-closed than in the eyes-open condition; (b) alpha power decreased with age for both the eyes-closed and eyes-open conditions; and (c) the alpha frequency was lower in older adults, showing a peak at $9 \mathrm{~Hz}$ compared to children and younger adults, whose peak was at $10 \mathrm{~Hz}$. Statistical analyses revealed significant age differences with respect to spectral alpha power, with children showing higher values than adults and younger adults higher values than older ones as indicated by post-hoc Fischer's LSD test (all $p s<.002$ ). The spectral alpha power was always lower for the eyes-open condition than for the eyes-closed condition, $F(1,106)=341.1$, $p<.0001, \eta^{2}=0.76$. There was also a significant Age by Eyes interaction, with the greatest reduction in alpha power (eyes-open compared to eyes-closed) observed in younger adults and the smallest reduction of alpha power observed in older adults. In addition, a significant Age by Electrodes interaction could be observed, indicating stronger age differences at parieto-occipital regions (see Table 1 and Figure 2 for details). (a)

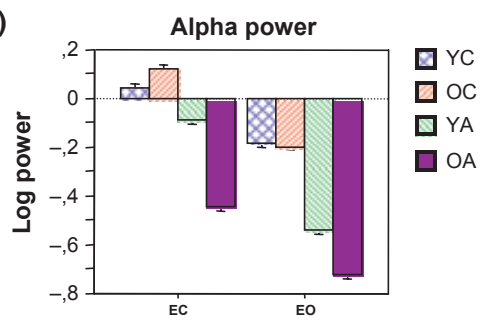

(b)


Figure 2 Lifespan differences in spectral power during the rest period. (a). Bar charts with mean values and standard error bars for alpha power for the eyes-closed (EC) and eyes-open (EO) conditions for the four age groups (YC, OC, YA, and OA). (b). Grand average frequency diagrams of spectral power at the Oz electrode for EC (blue curve) and EO (red curve) and corresponding brain maps for spectral power at the frequency peaks by age group. Note that the brain maps use the same scaling. Spectral power at the peak decreases with advancing age both in EC and EO conditions. 

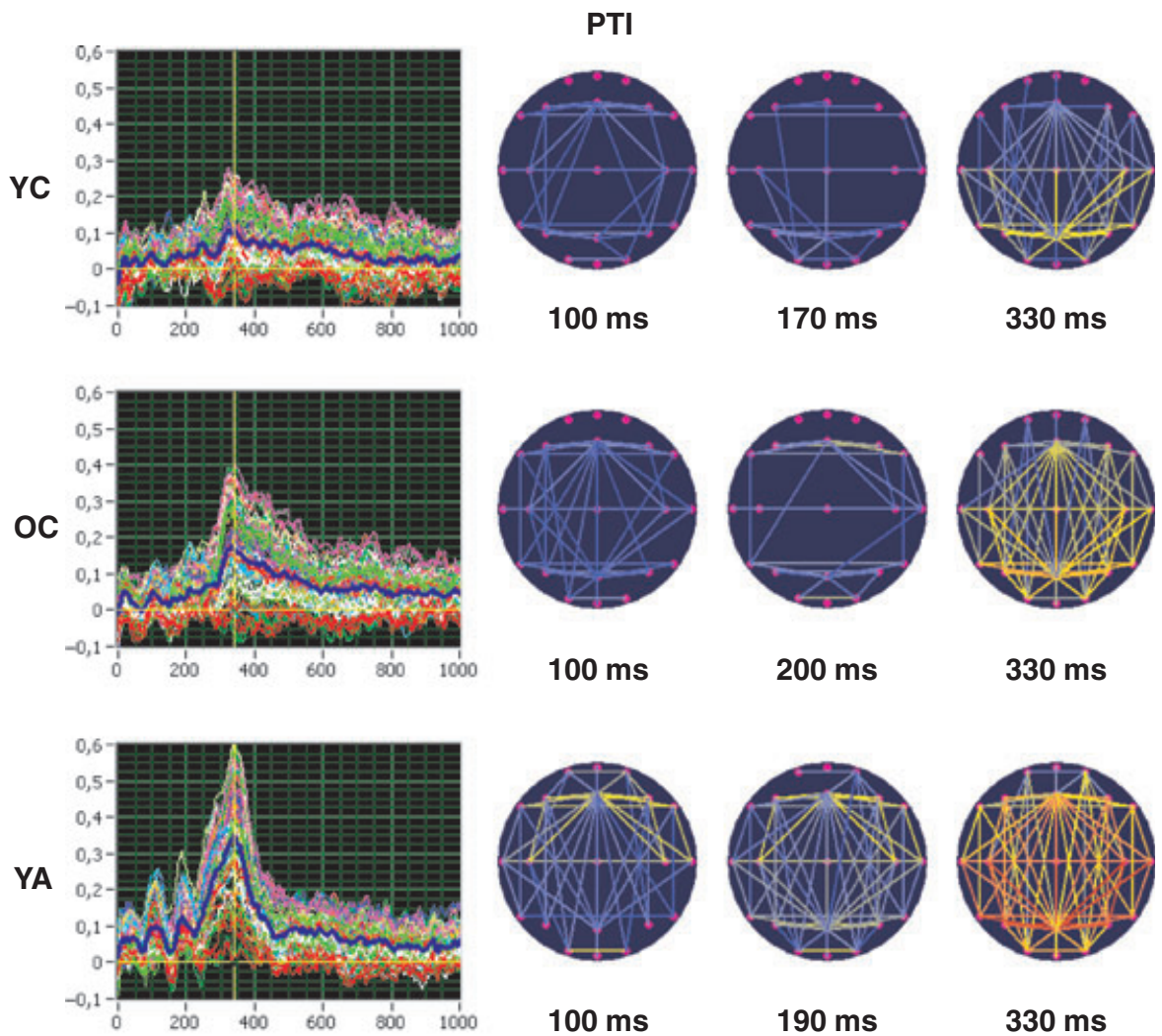

$100 \mathrm{~ms}$

$190 \mathrm{~ms}$

$330 \mathrm{~ms}$
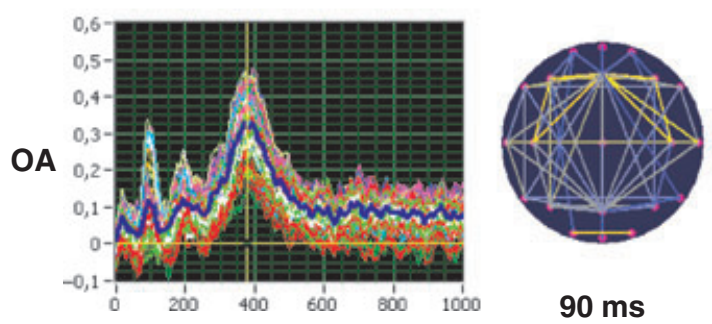

$90 \mathrm{~ms}$
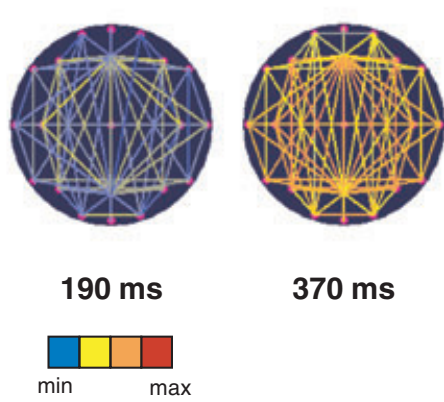

Figure 3 Lifespan differences in PTI during the task. Grand average stimulus-locked time diagrams of PTI difference values (task condition - rest condition) at the 76 electrode pairs for deviant stimuli under the attended condition by age group (YC, OC, YA, and $O A)$. Brain maps with the PTI connections at the three peaks are also shown. The strength of connections is indicated by color. $A$ strong increase in PTI with age, especially during the third peak, can be observed.

Lifespan differences in EEG dynamics during the oddball task

We determined complexity and coupling measures for standard and deviant stimuli during the passive listening (unattended) and active counting (attended) conditions. Age differences were most pronounced for deviant stimuli in the attended condition. Therefore, only results from this condition are considered here. We computed differences between measures obtained in the task and rest conditions (both with eyes closed) for statistical analyses and visualization. Grand averages of PTI differences (task condition - rest condition with eyes closed) across trials and participants stratified by age group are shown in Figure 3, which includes time diagrams for 76 electrode pairs and corresponding brain maps during three time intervals. Three positive PTI peaks during the time intervals of 100,180 and $330 \mathrm{~ms}$ can also be seen. The last peak was delayed by about $40 \mathrm{~ms}$ in older adults.

The PTI difference values were aggregated into seven different topological component scores and averaged around the three peaks (see the Methods section for details). These averages were then subjected to a threeway repeated measures ANOVA (Age $\times$ Topological Components $\times$ Peaks), which revealed a significant main effect for the Age factor, with significant differences being obtained between children and adults determined by Fischer's LSD post-hoc tests (OA, YA > YC, OC, all $p<.01)$. The significant main effects of Topological 



$90 \mathrm{~ms}$

$150 \mathrm{~ms}$

$330 \mathrm{~ms}$
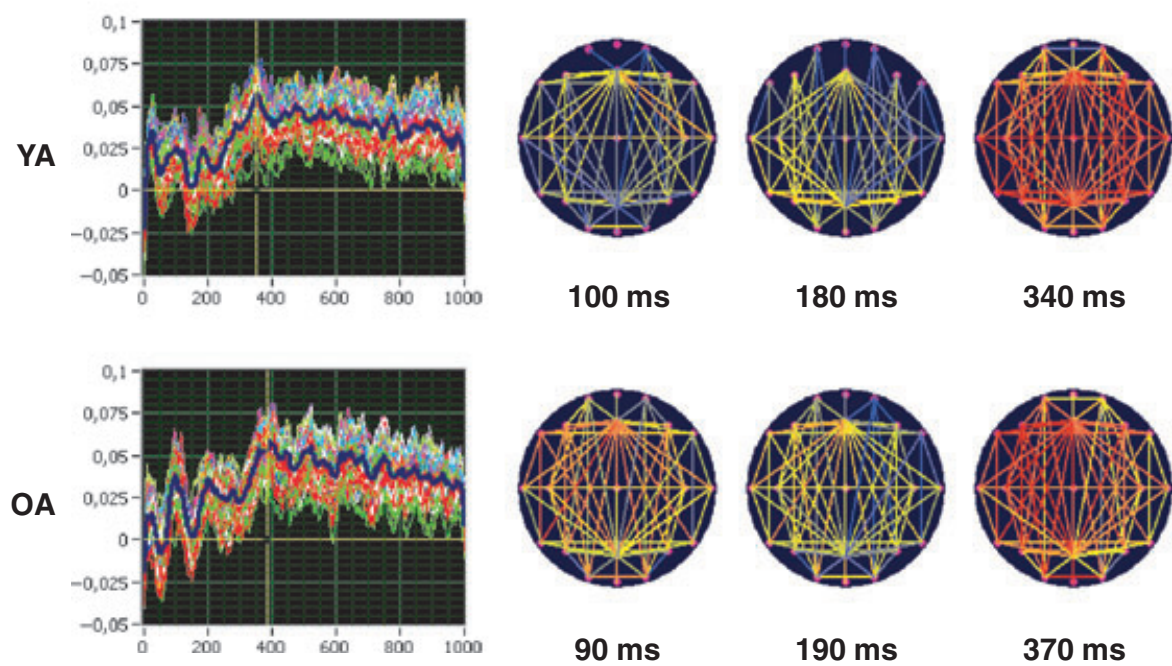

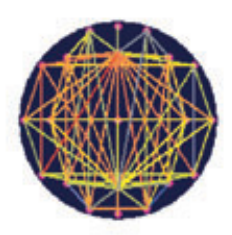

$90 \mathrm{~ms}$
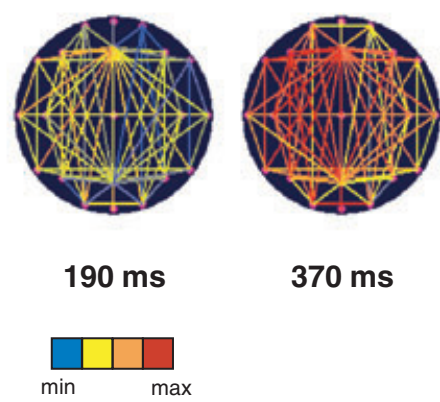

Figure 4 Lifespan differences in PCCD during the task. Grand average stimulus-locked time diagrams of PCCD difference values (task condition - rest condition) at the 76 electrode pairs for deviant stimuli under the attended condition by age group (YC, $O C, Y A$, and OA). Brain maps with the PCCD connections at the three peaks are also shown. The strength of the connections is indicated by color. A strong increase in PCCD with age, especially during the third peak, can be also observed.

Components, $F(6,636)=35.2, p<.0001, \eta^{2}=0.25$, and Peaks, $F(2,212)=71.8, p<.0001, \eta^{2}=0.40$, indicated higher nonlinear coupling for the LR (left-right) component and for the third coupling peak (in the time interval around $330 \mathrm{~ms}$ ). As demonstrated by the significant interactions Age by Topological Components, Age by Peaks and Age by Topological Components by Peaks, age differences were more marked at the topological components LR (left-right), WL (within left) and WR (within right), as well as being more marked for the third peak than for the other two peaks, again for the latter three topological components.

The grand averages of PCCD difference values across trials and participants in the four age groups are shown in Figure 4. As in the case of PTI, three positive PCCD peaks (at least for younger and older adults) during the time intervals of 100,180 and $330 \mathrm{~ms}$ can be seen, although the dynamics of this coupling differ somewhat from those found for PTI. For example, the third peak decreases very slowly and remains noticeable until the end of the trial. The last peak for older adults was once again delayed by about $30-40 \mathrm{~ms}$.

The PCCD difference values (in analogy to PTI) were combined in seven different topological components and averaged around the three peaks. A three-way repeated measures ANOVA (Age $\times$ Topological Components $\times$ Peaks) revealed a significant main effect for the Age factor, with significant differences between children and older adults only $(\mathrm{OA}>\mathrm{YC}$, OC, all $p<.05)$. Younger adults showed only marginally significant increased 
Table 2 ANOVA results ( $F$ - and p-values) for non-linear measures (PTI, PCCD and PD2) during task condition

\begin{tabular}{|c|c|c|c|c|}
\hline \multirow{2}{*}{$\frac{\text { Measures }}{\text { Coupling }}$} & \multicolumn{4}{|c|}{ Age-related effects and interactions } \\
\hline & Age $(\mathrm{df}=3,106)$ & Age $\times$ Peaks $(\mathrm{df}=6,212)$ & Age $\times$ TC $(\mathrm{df}=18,636)$ & Age $\times$ Peaks $\times$ TC $($ df $=36,1272)$ \\
\hline PCCD & $\begin{array}{l}3.3\left(p<.05, \eta^{2}=0.24\right) \\
\text { YC,OC< OA }\end{array}$ & $2.2\left(\mathrm{p}<.05, \eta^{2}=0.060\right)$ & $1.8(n s)$ & $1.8\left(p<.01, \eta^{2}=0.049\right)$ \\
\hline Complexity & Age $(\mathrm{df}=3,106)$ & Age $\times$ Peaks $(\mathrm{df}=6,212)$ & Age $\times$ Eld $(\mathrm{df}=18,636)$ & Age $\times$ Peaks $\times$ Eld $($ df $=120,4240)$ \\
\hline PD2 & $1.6(n s)$ & $2.4\left(p<.05, \eta^{2}=0.063\right)$ & $1.2(n s)$ & $1.2(n s)$ \\
\hline
\end{tabular}

Note: $n s=$ not significant $; \mathrm{TC}=$ Topological Components.

PCCD compared to younger children, Mean Diff. = 0.014 , Crit. Diff. $=0.015, p=.069$. The significant main effects of Topological Components, $F(6,636)=14.0$, $p<.0001, \eta^{2}=0.12$, and Peaks, $F(2,212)=42.9$, $p<.0001, \eta^{2}=0.29$, indicated higher nonlinear coupling for the LR (left-right) component and for the third coupling peak (in the time interval around $330 \mathrm{~ms}$ ). As shown by the significant interactions Age by Topological Components, Age by Peaks and Age by Topological Components by Peaks (see Table 2), age differences were more marked at the topological components LR (leftright), WL (within left), WR (within right) and $\mathrm{FzO}(\mathrm{Fz}$ to all other electrodes), as well as being more marked for the third peak, especially for these four topological components.

Dimensional complexity as measured by PD2 is displayed in Figure 5. We calculated difference values between the task and rest conditions with eyes closed for each of the 21 electrode locations. Complexity reduction related to stimulus processing at all three peaks was most pronounced at frontal and parietal sites. Statistical analyses showed significant main effects for Peaks and Electrodes, indicating a stronger reduction in complexity for the third peak and for the frontal and parietal electrode locations. The main effect of Age was not statistically reliable. However, there was a significant Age by Peaks interaction, showing more marked age-related differences for the third peak. Separate two-way repeated measures ANOVA (Age $\times$ Electrodes) for PD2-values at the third peak showed a significant main effect for the Age factor, $F(3,106)=2.7, p<.05, \eta^{2}=0.071$, with a significantly greater reduction in complexity in younger adults than younger children as indicated by the post-hoc Fischer's LSD test, Mean Diff. $=0.20$, Crit. Diff. $=0.14$, $p<.01$. We also observed a tendency for differences between younger children and older adults, Mean Diff. $=0.13$, Crit. Diff. $=0.14, p=.090$, and older children and younger adults, Mean Diff. $=0.12$, Crit. Diff. $=0.14, p=.075$.

\section{Correlations between nonlinear measures and independently assessed measures of perceptual speed}

To assess the functional status of the nonlinear measures obtained during the task, we correlated them with scores of the three independently assessed measures of percep- tual speed: Digit Letter Substitution (DLS), Digit Symbol Substitution (DSS), and Identical Pictures (IP). These three measures were $z$-transformed and combined by averaging to one overall measure of perceptual speed separately for each age group. In the case of nonlinear measures, we used difference values between the task and rest conditions from the third peak only, as it was the most pronounced and also showed the most marked agerelated effects. We expected perceptual speed scores to be positively related to coupling measures and negatively related to the complexity measure. Correlations were reliable for adults but not for children, with the exception of a significant correlation in OC for the PD2 measure at F7 electrode, $r=-0.41, p=.028$, indicating that a stronger decrease in dynamic complexity is related to a higher score for perceptual speed. Although only one correlation coefficient was significantly different from 0 , the other correlation coefficients, with the exception of one electrode, were negative. The sign test showed a highly significant tendency for a negative relationship $(p<.0001)$. The highest correlations were observed for the PTI measure, particularly in older adults (see Table 3 for details). Younger adults showed reliable positive correlations between the perceptual speed score and three PTI components on the anterior-posterior axis: AP, $\mathrm{PA}$, and $\mathrm{PzO}$. In the case of the PCCD measure, there were some correlations in younger adults, again concerning the anterior-posterior axis, i.e. AP and $\mathrm{PzO}$, whereas correlations in older adults were related to connections within the left (WL), and between left and right hemispheres (LR). Due to the fact that all correlations in both adult groups were positive, the sign test showed a correspondingly highly significant tendency $(p=.0078)$ for the positive relationship in these cases. Apart from the correlation in OC reported above, the correlations between perceptual speed and PD2 were only reliable in older adults at the two parieto-occipital electrodes, Pz: $r=-0.39, p=.042, \quad \mathrm{O} 2: \quad r=-0.45$, $p=.016$. Again, the sign test showed a highly significant tendency $(p<.0001)$ for the negative relationship between PD2 and perceptual speed scores. These significant negative correlations indicate that, among older adults, higher perceptual speed is related to a more marked reduction in complexity during the third peak of target stimulus processing. We need to emphasize that the reported associations between brain dynamics and 



Figure 5 Lifespan differences in PD2 during the task. Grand average stimulus-locked time diagrams of PD2 difference values (task condition - rest condition) at the 21 electrodes for deviant stimuli under the attended condition by age group (YC, OC, YA, and OA). Brain maps with topological distribution of PD2 values at the three peaks are shown. A strong PD2 or complexity reduction with age, especially during the third peak, can be observed.

perceptual speed performance were observed for a few electrodes only, and that alpha levels were not corrected for multiple comparisons. Hence, the observed correlations may be accidental and need to be replicated in independent samples. Note, however, that the signs of the correlations followed a clear pattern.

\section{Discussion}

We examined age-group differences in nonlinear brain dynamics during rest and auditory oddball performance. The main findings are that: (a) the assumption that the empirically observed EEG time series are generated by a nonlinear dynamic system and are nonlinear in all age groups is tenable; (b) nonlinear coupling measures and alpha power both decrease during rest with eyes open compared to rest with eyes closed; complexity, by contrast, increases; (c) dimensional complexity in the rest conditions increases from childhood to old age, whereas nonlinear coupling decreases; these changes in dynamic complexity and nonlinear coupling were accompanied by a decrease in alpha power with age; and (d) the increase in nonlinear coupling during auditory oddball performance is accompanied by complexity reduction, again with pronounced differences observed as a function of age group. Agegroup differences also varied by rest condition, processing stage, and electrode site or topological coupling component. 
Table 3 Correlation coefficients for non-linear coupling measures (PTI and PCCD) with an integrative measure of perceptual speed during the oddball task condition in younger and older adults

\begin{tabular}{llllll}
\hline & \multicolumn{2}{c}{ Younger adults } & & \multicolumn{2}{c}{ Older adults } \\
\cline { 2 - 3 } \cline { 5 - 6 } Components & PTI & PCCD & & PTI & PCCD \\
\hline AP & $0.40^{*}$ & $0.36^{*}$ & & 0.34 & 0.21 \\
PA & $0.37^{*}$ & 0.26 & & 0.31 & 0.20 \\
LR & 0.33 & 0.33 & & $0.54^{* *}$ & $0.39^{*}$ \\
WL & 0.27 & 0.34 & & $0.53^{* *}$ & $0.39^{*}$ \\
WR & 0.25 & 0.21 & & $0.40^{*}$ & 0.26 \\
FzO & 0.35 & 0.29 & & $0.45^{*}$ & 0.31 \\
PzO & $0.37^{*}$ & $0.36^{*}$ & & $0.44^{*}$ & 0.25 \\
\hline
\end{tabular}

Note: ${ }^{*} p<.05 ;{ }^{* *} p<.01$. AP $=$ anterior to posterior, $\mathrm{PA}=$ posterior to anterior, $\mathrm{LR}=$ left to right (or right to left), $\mathrm{WL}=$ within left, $\mathrm{WR}=$ within right, $\mathrm{FzO}=\mathrm{Fz}$ to all other electrode locations, and $\mathrm{PzO}=\mathrm{Pz}$ to all other electrode locations.

An increase in complexity in resting conditions with eyes open compared with eyes closed has been previously observed in other studies in adults (Mayer-Kress \& Layne, 1987; Müller et al., 2003a; Rapp et al., 1989; Stam, Tavy, Jelles, Achtereekte, Slaets \& Keunen, 1994). Here, we showed that these complexity changes also occur in children and older adults, and that they are more pronounced in adults than in children. Furthermore, in all age groups, the increase in dynamic complexity was also accompanied by a decrease in nonlinear coupling and spectral alpha power. Decreases in nonlinear coupling in the eyes-open compared to the eyes-closed condition had never been investigated before and thus represent a new finding, indicating that a loss in dynamic coupling occurs through opening the eyes. These results are difficult to explain by volume conduction as an artificial coupling source, as this mechanism would have preferentially enhanced coupling between closer electrode sites (Nunez \& Srinivasan, 2006). However, many of the couplings observed here involve electrode pairs $6-8 \mathrm{~cm}$ apart or further. Most importantly, it is difficult to imagine how volume conductance effects would differ by task condition.

Decreases in spectral alpha power (also called alpha depression) during rest with eyes open compared to rest with eyes closed are a well-known phenomenon (see Klimesch, 1999, for a review). Alpha reactivity increases from childhood to early adulthood (Klimesch, 1999; Somsen, van't Klooster, van der Molen, van Leeuwen \& Licht, 1997), and decreases thereafter (Duffy, Albert, McAnulty \& Garvey, 1984; Shaw, 2003). In our study, we observed a significant Age by Eyes interaction, indicating age-related differences in alpha reactivity, which was highest in younger adults and lowest in younger children and older adults. Normally, alpha depression in the eyesopen condition is associated with brain activation caused by increased external stimulation through the opening of the eyes or visual input (Klimesch, 1999). Thus, increases in complexity and decreases in coupling are related to increased levels of brain activation that also differ with age, as shown by the Age by Eyes interaction.

According to previous research, increases in complexity are associated with an increasing number of simultaneously active cell assemblies (Elbert et al., 1994; Lutzenberger et al., 1995), and a decrease in the coupling signals loss in neural connections between some parts of the system. It thus seems that some larger cell assemblies active in the eyes-closed condition disintegrate when the eyes are opened into a higher number of cell assemblies (higher complexity). At the same time, some connections between neural elements are lost (lower coupling). As expected, these dynamic effects appear to increase with age.

As shown in computational studies (Deco et al., 2008, 2009; Ghosh et al., 2008), the intrinsic noise given by the variance of the stochastic fluctuations is a driving property of larger scale resting state networks. This noise effect also includes stochastic resonance emerging from optimal levels of noise, which is essential for the expression of spatiotemporal synchronization patterns. $\mathrm{Li}$, von Oertzen and Lindenberger (2006) suggested that brain aging is associated with higher internal noise and reduced peak stochastic resonance. This could be a reason for the increasing complexity of EEG time series and reduced nonlinear coupling during resting state with increasing age. Recently, it was found (McIntosh et al., 2010) that brain noise or variability of the signal increases with maturation and also with aging. Authors suggested that brain noise is generated by the deterministic and random components of the brain networks, and that its changes represent the enhancement of functional network potential - the brain's dynamic repertoire. Whether these changes in older adults are more determined by the deterministic or random (stochastic) components of the brain networks remains to be seen.

We also found a strong increase in nonlinear coupling and a decrease in dimensional complexity during task performance relative to the resting condition. These changes were most pronounced in the time interval of $330-370 \mathrm{~ms}$, when stimulus processing presumably demanded the most resources. As mentioned above, complexity reduction during stimulus processing was also found in other studies using pointwise complexity measures (Molnar et al., 1995; Rapp et al., 1989). To our knowledge, the combination of complexity reduction with strong nonlinear coupling has been observed here for the first time. In principle, the fact that an increase in synchronization or coupling occurs during stimulus processing is, in itself, not really new (Singer, Engel, Kreiter, Munk, Neuenschwander \& Roelfsema, 1997; Varela, Lachaux, Rodriguez \& Martinerie, 2001), and is also consistent with the results of an earlier analysis with phase synchronization and/or phase coherence measures (Müller et al., 2009). However, in this study, we were able to show that the reduction in complexity and the increase in nonlinear coupling have a similar time course, perhaps reflecting the same set of neural mechanisms during 
stimulus processing. Complexity reduction during stimulus processing could be due to a loss in the number of degrees of freedom and may therefore be related to decision-making (Anokhin, 1974). The loss of degrees of freedom in the system is apparently related to a decrease in the number of cell assemblies involved in stimulus processing. At the same time, we observed a clear increase in nonlinear coupling, indicating stronger connections or higher levels of information exchange within and between cell assemblies. This could possibly reflect the fusion of initially independent cell assemblies into larger such assemblies and to a corresponding reduction in the total number of cell assemblies.

Furthermore, we were able to show that EEG complexity and nonlinear couplings undergo profound changes from childhood to adulthood and old age, with strong increases in nonlinear coupling and complexity reduction related to stimulus processing. The most pronounced age differences were found in nonlinear coupling, especially as measured by PTI as an index of the information content of nonlinear coupling dynamics. Interestingly, this measure also showed the most prominent correlation with a behavioral marker of perceptual speed. The corresponding correlations were reliably positive in adult age groups but not in children. In our previous study (Müller et al., 2009), we found significant positive correlations between perceptual speed in younger adults as measured by IP (Identical Pictures) and two synchronization measures, namely the Phase Locking Index (PLI) and Evoked Power (EP); the same correlations were, however, negative in older adults. In this study, the correlations with PTI (and also with PCCD) were positive in the both groups. The positive correlation of the two nonlinear coupling measures, PTI and PCCD, with perceptual speed indicates that higher nonlinear coupling to the stimulus during auditory oddball performance was related to faster performance on separately assessed measures of perceptual speed. Thus, in contrast to linear coupling (phase synchronization; cf. Müller et al., 2009), the relation between nonlinear coupling and cognitive performance remained positive throughout the entire adult lifespan. Given that all of the correlations were positive in sign, and that most of them were statistically reliable, we are confident that the observed associations between brain dynamics and behavioral performance were not accidental. Furthermore, this positive relationship was also confirmed by the sign test. We also note that PTI and PCCD have been introduced into the literature only recently. Both measures are intended to capture mutual interaction of subsystems evolving in multidimensional phase space. The PTI describes the dependencies of the trajectories reconstructed in the phase space, and the PCCD quantifies dissipative coupling dynamics (Vandenhouten, 1998). This study is among the first to explore the associations of these two measures to behavior. Clearly, the qualitative and quantitative properties of these measures, including their convergent and divergent validity, await further scrutiny.
Future research is needed to elaborate the theoretical significance of the results obtained (see also Deco et al., 2008), especially in relation to age-graded changes in brain dynamics across the lifespan (e.g. Mcintosh et al., 2010).

In our previous study (Müller et al., 2009), we suggested that the inverse relations between age trends in phase synchronization and spectral power may indicate a lifespan shift from rate coding in children to temporal coding in adults. Whereas the cognitive system in children may prevalently use a rate code, where the rate of nerve impulses over time codes for sensory and motor events (Stein, Gossen \& Jones, 2005), the cognitive systems of adults may be more likely to use 'temporal coding', which requires the exact timing of neural spikes (Hestrin \& Galarreta, 2005; Mehta, Lee \& Wilson, 2002). Synaptic plasticity or Hebbian learning plays a crucial role in information processing and involves synaptic changes ranging from short-term plasticity or short-term potentiation (STP), indicating changes that occur in milliseconds up to minutes, to long-term plasticity or long-term potentiation (LTP), which occur within a timescale of hours or longer (Abbott \& Regehr, 2004). Synaptic connections are altered by temporal spatial firing patterns of pre-synaptic neurons (Abbott \& Regehr, 2004; Destexhe \& Marder, 2004). In other words, firing strength is dependent on temporal tuning between pre-synaptic and post-synaptic neurons, suggesting that temporal coding can involve different synaptic signal transmission mechanisms. Increased reductions in complexity and more marked increases in nonlinear coupling in adults than in children during stimulus processing, which presumably indicate more flexible changes in the number of active cell assemblies and the strength of connections between neural elements, are in line with our hypothesis that there is a lifespan shift with regard to coding, moving from rate coding in children to temporal coding in adults, as the rate code is less flexible than the temporal code.

In sum, the present study shows that cortical dynamics undergo profound changes from childhood to adulthood and old age. We suggest that the changes observed in the resting states and during task performance reflect basic mechanisms relating to the organization of the cell assemblies and their functional reorganization across the lifespan. More research is needed to understand the ways in which maturational changes in the brain's dynamic complexity and coupling are shaped by neurochemical, neuroanatomic, and neurofunctional mechanisms, and to judge the extent to which changes in nonlinear dynamics are related to changes in cognitive processes. In addition, it seems worth exploring whether the methods and findings reported here may inform the development of diagnostic techniques for dementia and other neurological abnormalities (Czigler et al., 2008; Jelles et al., 1999; Jeong, 2004; Müller, Lutzenberger, Pulvermüller, Mohr \& Birbaumer, 2001).

Finally, some limitations of the present study need to be acknowledged. First, due to the comparatively small 
sample size, the present analyses may have failed to identify some group differences in nonlinear dynamics that do in fact exist. Second, only two different age groups of children participated in this study. Future research will be needed to examine age differences at earlier and also later periods of childhood as well as in very old age, and to trace the development of nonlinear dynamics longitudinally across extended periods of the human lifespan.

\section{Acknowledgements}

This research was financially supported by grant FOR 448 from the Deutsche Forschungsgemeinschaft and by the Max Planck Society. The authors thank Yvonne Brehmer for contributing to the design of the study and for supervising data collection; Timo von Oertzen and Zurab Schera for programming and hardware assistance; Roland Benoit, Stephan Glied, Esther Runkel, Myriam Sander, Gundula Stoll, and Benjamin Straube for testing the research participants; and Julia Delius for language assistance.

\section{References}

Abbott, L.F., \& Regehr, W.G. (2004). Synaptic computation. Nature, 431, 796-803. doi:10.1038/nature03010

Anokhin, A.P., Birbaumer, N., Lutzenberger, W., Nikolaev, A., \& Vogel, F. (1996). Age increases brain complexity. Electroencephalography and Clinical Neurophysiology, 99, 6368. doi:10.1016/0921-884X(96)95573-3

Anokhin, A.P., Lutzenberger, W., \& Birbaumer, N. (1999). Spatiotemporal organization of brain dynamics and intelligence: an EEG study in adolescents. International Journal of Psychophysiology, 33, 259-273. doi:10.1016/S01678760(99)00064-1

Anokhin, A.P., Lutzenberger, W., Nikolaev, A., \& Birbaumer, N. (2000). Complexity of electrocortical dynamics in children: developmental aspects. Developmental Psychobiology, 36, 9-22. doi:10.1002/(SICI)1098-2302(200001)36:1 < 9:: AID-DEV2 > 3.0.CO;2-5

Anokhin, A.P., Muller, V., Lindenberger, U., Heath, A.C., \& Myers, E. (2006). Genetic influences on dynamic complexity of brain oscillations. Neuroscience Letters, 397, 93-98. doi:10.1016/j.neulet.2005.12.025

Anokhin, P.K. (1974). Biology and neurophysiology of the condition reflex and its role in adaptive behavior. Oxford: Pergamon Press.

Ashby, W.R. (1958). Requisite variety and implications for control of complex systems. Cybernetica, 1, 83-99.

Baltes, P.B., \& Lindenberger, U. (1997). Emergence of a powerful connection between sensory and cognitive functions across the adult life span: a new window to the study of cognitive aging? Psychology \& Aging, 12, 12-21. doi:10.1037/ 0882-7974.12.1.12

Baltes, P.B., \& Mayer, K.U. (Eds.) (1999). The Berlin Aging Study: Aging from 70 to 100. New York: Cambridge University Press.
Birbaumer, N., Flor, H., Lutzenberger, W., \& Elbert, T. (1995). Chaos and order in the human brain. Electroencephalography and Clinical Neurophysiology Supplement, 44, 450-459.

Biswal, B., Yetkin, F.Z., Haughton, V.M., \& Hyde, J.S. (1995). Functional connectivity in the motor cortex of resting human brain using echo-planar MRI. Magnetic Resonance in Medicine, 34, 537-541. doi:10.1002/mrm. 1910340409

Boker, S.M., Molenaar, P.C., \& Nesselroade, J.R. (2009). Issues in intraindividual variability: individual differences in equilibria and dynamics over multiple time scales. Psychology \& Aging, 24, 858-862. doi:10.1037/a0017912

Briggs, K. (1990). An improved method for estimating Lyapunov exponents of chaotic time series. Physics Letters A, 151, 27-32. doi:10.1016/0375-9601(90)90841-B

Buzsáki, G. (2006). Rhythms of the brain. New York: Oxford University Press.

Carhart, R., \& Jerger, J.F. (1959). Preferred method for clinical determination of pure-tone thresholds. Journal of Speech and Hearing Disorders, 24, 330-345.

Czigler, B., Csikós, D., Hidasi, Z., Gaál, Z.A., Csibri, E., Kiss, É., Salacz, P., \& Molnár, M. (2008). Quantitative EEG in early Alzheimer's disease patients: power spectrum and complexity features. International Journal of Psychophysiology, 68, 75-80.

Damoiseaux, J.S., Rombouts, S.A., Barkhof, F., Scheltens, P., Stam, C.J., Smith, S.M., \& Beckmann, C.F. (2006). Consistent resting-state networks across healthy subjects. Proceedings of the National Academy of Sciences, USA, 103, 13848-13853. doi:10.1073/pnas.0601417103

Deco, G., Jirsa, V.K., Robinson, P.A., Breakspear, M., \& Friston, K. (2008). The dynamic brain: from spiking neurons to neural masses and cortical fields. PLoS Computational Biology, 4, e1000092. doi:10.1371/journal.pcbi.1000092

Deco, G., Jirsa, V., McIntosh, A.R., Sporns, O., \& Kotter, R. (2009). Key role of coupling, delay, and noise in resting brain fluctuations. Proceedings of the National Academy of Sciences, USA, 106, 10302-10307. doi:10.1073/pnas.0901831106

Destexhe, A., \& Marder, E. (2004). Plasticity in single neuron and circuit computations. Nature, 431, 789-795.

Duffy, F.H., Albert, M.S., McAnulty, G., \& Garvey, A.J. (1984). Age-related differences in brain electrical activity of healthy subjects. Annals of Neurology, 16, 430-438. doi:10.1002/ana.410160403

Eckmann, J.P., \& Ruelle, D. (1985). Ergodic theory of chaos and strange attractors. Reviews of Modern Physics, 57, 617 656. doi:10.1103/RevModPhys.57.617

Elbert, T., Ray, W.J., Kowalik, Z.J., Skinner, J.E., Graf, K.E., \& Birbaumer, N. (1994). Chaos and physiology: deterministic chaos in excitable cell assemblies. Physiological Reviews, 74, $1-47$.

Ellner, S. (1988). Estimating attractor dimensions from limited data: a new method, with error estimates. Physics Letters A, 133, 128-133. doi:10.1016/0375-9601(88)90772-4

Ghosh, A., Rho, Y., McIntosh, A.R., Kotter, R., \& Jirsa, V.K. (2008). Noise during rest enables the exploration of the brain's dynamic repertoire. PLoS Computational Biology, 4, e1000196. doi:10.1371/journal.pcbi.1000196

Grassberger, P., \& Procaccia, I. (1983). Measuring the strangeness of strange attractors. Physica, 9D, 189-208. 
Gratton, G., Coles, M.G., \& Donchin, E. (1983). A new method for off-line removal of ocular artifact. Electroencephalography and Clinical Neurophysiology, 55, 468-484.

Greicius, M.D., Krasnow, B., Reiss, A.L., \& Menon, V. (2003). Functional connectivity in the resting brain: a network analysis of the default mode hypothesis. Proceedings of the National Academy of Sciences, USA, 100, 253-258. doi:10.1073/pnas.0135058100

Hebb, D.O. (1949). The organization of behavior: A neuropsychological theory. New York: Wiley \& Sons.

Hestrin, S., \& Galarreta, M. (2005). Synchronous versus asynchronous transmitter release: a tale of two types of inhibitory neurons. Nature Neuroscience, 8, 1283-1284.

Jelles, B., van Birgelen, J.H., Slaets, J.P., Hekster, R.E., Jonkman, E.J., \& Stam, C.J. (1999). Decrease of non-linear structure in the EEG of Alzheimer patients compared to healthy controls, Clinical Neurophysiology, 110, 1159-1167. doi: 10.1016/S1388-2457(99)00013-9

Jeong, J. (2004). EEG dynamics in patients with Alzheimer's disease. Clinical Neurophysiology, 115, 1490-1505. doi: 10.1016/j.clinph.2004.01.001

Klimesch, W. (1999). EEG alpha and theta oscillations reflect cognitive and memory performance: a review and analysis, Brain Research Review, 29, 169-195. doi:10.1016/S01650173(98)00056-3

Lambertz, M., Vandenhouten, R., Grebe, R., \& Langhorst, P. (2000). Phase transitions in the common brainstem and related systems investigated by nonstationary time series analysis. Journal of the Autonomic Nervous System, 78, 141157. doi:10.1016/S0165-1838(99)00072-7

Li, S.-C., Lindenberger, U., \& Sikstrom, S. (2001). Aging cognition: from neuromodulation to representation. Trends in Cognitive Sciences, 5, 479-486. doi:10.1016/S13646613(00)01769-1

Li, S.-C., von Oertzen, T., \& Lindenberger, U. (2006). A neurocomputational model of stochastic resonance and aging. Neurocomputing, 69, 1553-1560. doi:10.1016/j.neucom.2005. 06.015

Lindenberger, U., Mayr, U., \& Kliegl, R. (1993). Speed and intelligence in old age. Psychology \& Aging, 8, 207-220. doi:10.1037/0882-7974.8.2.207

Lutzenberger, W., Preissl, H., \& Pulvermuller, F. (1995). Fractal dimension of electroencephalographic time series and underlying brain processes. Biological Cybernetics, 73, 477482. doi:10.1007/BF00201482

Mayer-Kress, G. (1998). Non-linear mechanisms in the brain. Zeitschrift für Naturforschung C, 53, 677-685.

Mayer-Kress, G., \& Layne, S.P. (1987). Dimensionality of the human electroencephalogram. In S.H. Koslow (Ed.), Perspectives in biological dynamics and theoretical medicine (Vol. 504, pp. 62-87). New York: New York Academy of Sciences.

McIntosh, A.R., Kovacevic, N., Lippe, S., Garrett, D., Grady, C., \& Jirsa, V. (2010). The development of a noisy brain. Archives Italiennes de Biologie, 148, 323-337.

Mehta, M.R., Lee, A.K., \& Wilson, M.A. (2002). Role of experience and oscillations in transforming a rate code into a temporal code. Nature, 417, 741-746. doi:10.1038/nature 00807

Molnar, M., Skinner, J.E., Csepe, V., Winkler, I., \& Karmos, G. (1995). Correlation dimension changes accompanying the occurrence of the mismatch negativity and the P3 event-re- lated potential component. Electroencephalography and Clinical Neurophysiology, 95, 118-126. doi:10.1016/00134694(95)00039-2

Müller, V., Birbaumer, N., Preissl, H., Braun, C., Mayer-Kress, G., \& Lang, F. (2003a). Effects of hydration and hyperventilation on cortical complexity. Experimental Brain Research, 150, 341-355. doi:10.1007/s00221-003-1425-5

Müller, V., Brehmer, Y., von Oertzen, T., Li, S.-C., \& Lindenberger, U. (2008). Electrophysiological correlates of selective attention: a lifespan comparison. BMC Neuroscience, 9, 18. doi:10.1186/1471-2202-9-18

Müller, V., Gruber, W., Klimesch, W., \& Lindenberger, U. (2009). Lifespan differences in cortical dynamics of auditory perception. Developmental Science, 12, 839-853. doi:10.1111/ j.1467-7687.2009.00834.x

Müller, V., Lutzenberger, W., Pulvermüller, F., Mohr, B., \& Birbaumer, N. (2001). Investigation of brain dynamics in Parkinson's disease by methods derived from nonlinear dynamics. Experimental Brain Research, 137, 103-110. doi: $10.1007 / \mathrm{s} 002210000638$

Müller, V., Preiß1, H., Lutzenberger, W., \& Birbaumer, N. (2003b). Komplexität und Hirndynamik. In G. Schiepek, (Ed.), Neurobiologie der Psychotherapie (pp. 58-79). Stuttgart and NewYork: Schattauer.

Nelson, C.A., \& Luciana, M. (Eds.) (2001). Handbook of developmental cognitive neuroscience. Cambridge, MA: MIT Press.

Nunez, P.L., \& Srinivasan, R. (2006). Electric fields of the brain: The neurophysics of EEG. Oxford and New York: Oxford University Press.

Park, J., Carp, J., Hebrank, A., Park, D.C., \& Polk, T.A. (2010). Neural specificity predicts fluid processing ability in older adults. Journal of Neuroscience, 30 (27), 9253-9259.

Park, D.C., Polk, T.A., Park, R., Minear, M., Savage, A., \& Smith, M.R. (2004). Aging reduces neural specialization in ventral visual cortex. Proceedings of the National Academy of Sciences, USA, 101, 13091-13095. doi:10.1073/pnas. 0405148101

Park, D.C., \& Reuter-Lorenz, P. (2009). The adaptive brain: aging and neurocognitive scaffolding. Annual Review of Psychology, 60, 173-196. doi:10.1146/annurev.psych.59. 103006.093656

Pierce, T.W., Kelly, S.P., Watson, T.D., Replogle, D., King, J.S., \& Pribram, K.H. (2000). Age differences in dynamic measures of EEG. Brain Topography, 13, 127-134. doi: 10.1023/A:1026659102713

Pierce, T.W., Watson, T.D., King, J.S., Kelly, S.P., \& Pribram, K.H. (2003). Age differences in factor analysis of EEG. Brain Topography, 16, 19-27. doi:10.1023/A:1025654331788

Pritchard, W.S., \& Duke, D.W. (1992). Measuring chaos in the brain: a tutorial review of nonlinear dynamical EEG analysis. International Journal of Neuroscience, 67, 31-80. doi:10.3109/ 00207459208994774

Rapp, P.E., Bashore, T.R., Martinerie, J.M., Albano, A.M., Zimmerman, I.D., \& Mees, A.I. (1989). Dynamics of brain electrical activity. Brain Topography, 2, 99-118. doi:10.1007/ BF01128848

Reuter-Lorenz, P.A., \& Park, D.C. (2010). Human neuroscience and the aging mind: a new look at old problems. Journal of Gerontology: Psychological Sciences, 65B (4), 405-415. doi:10.1093/geronb/gbq035

Shannon, C.E., \& Weaver, W. (1949). The mathematical theory of communication. Urbana, IL: The University of Illinois Press. 
Shaw, J.C. (2003). The brain's alpha rhythms and the mind. Amsterdam: Elsevier.

Singer, W., Engel, A.K., Kreiter, A.K., Munk, M.H.J., Neuenschwander, S., \& Roelfsema, P.R. (1997). Neuronal assemblies: necessity, signature, and detectability. Trends in Cognitive Sciences, 1, 252-261. doi:10.1016/S13646613(97)01079-6

Skinner, J.E., Molnar, M., Vybiral, T., \& Mitra, M. (1992). Application of chaos theory to biology and medicine. Integrative Physiological and Behavioral Science, 27, 39-53. doi:10.1007/BF02691091

Skinner, J.E., Pratt, C.M., \& Vybiral, T. (1993). A reduction in the correlation dimension of heartbeat intervals precedes imminent ventricular fibrillation in human subjects. American Heart Journal, 125, 731-743. doi:10.1016/00028703(93)90165-6

Somsen, R.J., van't Klooster, B.J., van der Molen, M.W., van Leeuwen, H.M., \& Licht, R. (1997). Growth spurts in brain maturation during middle childhood as indexed by EEG power spectra. Biological Psychology, 44, 187-209. doi:10.1016/S0301-0511(96)05218-0

Stam, C.J. (2005). Nonlinear dynamical analysis of EEG and MEG: review of an emerging field, Clinical Neurophysiology, 116, 2266-2301. doi:10.1016/j.clinph.2005.06.011

Stam, K.J., Tavy, D.L., Jelles, B., Achtereekte, H.A., Slaets, J.P., \& Keunen, R.W. (1994). Non-linear dynamical analysis of multichannel EEG: clinical applications in dementia and Parkinson's disease. Brain Topography, 7, 141-150. doi:10.1007/BF01186772

Stein, R.B., Gossen, E.R., \& Jones, K.E. (2005). Neuronal variability: noise or part of the signal? Nature Reviews Neuroscience, 6, 389-397. doi:10.1038/nrn1668

Takens, F. (1981). Detecting strange attractors in turbulence. Lecture notes in mathematics (Vol. 898, pp. 366-81). Berlin: Springer.
Theiler, J., Eubank, S., Longtin, A., Galdrikian, B., \& Farmer, J.D. (1992). Testing for nonlinearity in time series: the method of surrogate data. Physica D, 58, 77-94.

Vaillancourt, D.E., \& Newell, K.M. (2002). Changing complexity in human behavior and physiology through aging and disease. Neurobiology of Aging, 23, 1-11.

Vandenhouten, R. (1998). Analyse instationärer Zeitreihen komplexer Systeme und Anwendung in der Physiologie. Aachen: Shaker.

van Drongelen, W. (2007). Signal processing for neuroscientists: Introduction to the analysis of physiological signals. London: Academic Press.

Varela, F., Lachaux, J.P., Rodriguez, E., \& Martinerie, J. (2001). The brainweb: phase synchronization and large-scale integration. Nature Reviews Neuroscience, 2, 229-239. doi:10.1038/35067550

Venables, N.C., Bernat, E.M., \& Sponheim, S.R. (2009). Genetic and disorder-specific aspects of resting state EEG abnormalities in schizophrenia. Schizophrenia Bulletin, 35, 826-839. doi:10.1093/schbul/sbn021

Watts, D. (2003). Six degrees: The science of a connected age. New York: W.W. Norton \& Company.

Wechsler, D. (1955). Wechsler Adult Intelligence Scale Manual. New York: Psychological Corporation.

Wolf, A., Swift, J.B., Swinney, H.L., \& Vastano, A. (1985). Determining Lyapunov exponents from a time series. Physica D, 16, 285-317. doi:10.1016/0167-2789(85)90011-9

Received: 25 May 2011

Accepted: 2 February 2012 